\title{
Patterns of enrollment in randomized and preference trials of behavioral treatments for insomnia
}

\author{
Souraya Sidani \\ Ryerson University \\ Patricia Moritz \\ University of Colorado \\ Health Sciences Center \\ J oyal Miranda \\ Université de Montréal
}

\author{
Richard R. Bootzin \\ University of Arizona \\ Dana R. Epstein \\ Phoenix Veterans Affairs \\ Health Care System \\ Jennifer Cousins \\ University of Pittsburgh Medical Center
}

\begin{abstract}
Participants' preferences for treatment may deter enrollment in a randomized clinical trial (RCT). The Partially randomized clinical trial (PRCT) is proposed as an alternative design to increase enrollment rate and enhance representativeness of the sample. There is limited evidence supporting the advantages of the PRCT. This study aimed to examine enrollment and refusal rates, reasons for refusal, and clinical profile of persons who declined participation and those who enrolled, in the context of a RCT and a PRCT. Persons with chronic insomnia completed a questionnaire to determine if they met the eligibility criteria regarding type, frequency, and duration of insomnia. Those who declined participation indicated reasons for refusal. Enrollment rate was computed as the percentage of individuals who took part in the study out of those found eligible. Independent sample t-test was used to compare enrollees and non-enrollees on characteristics of insomnia. The results showed a higher enrollment rate in the RCT than PRCT. Reasons for refusal were similar under the RCT and PRCT. Significant differences between enrollees and non-enrollees were found on fewer characteristics in the RCT than PRCT. The results do not support the advantages of the PRCT in enhancing enrollment of participants in studies evaluating the effectiveness of behavioral treatments of chronic insomnia.
\end{abstract}

Keywords: preference trials, randomized clinical trial, enrollment, chronic insomnia, behavioral treatment

The validity of conclusions reached in intervention evaluation research is contingent on, among other factors, enrolling an adequate number of participants who are representative of the target population. Sample size is a major determinant of the study statistical power. Accrual of the sample size required on the basis of power analysis is a safeguard against type II error (Harris \& Dyson, 2001). Representativeness of the sample is critical for external validity. The socio-demographic and clinical characteristics of the sample should be comparable to those of the target population in order to enhance the generalizability of the findings. This comparability implies 
that participants should be similar to non-participants on baseline characteristics, as non-participants are also members of the target population (Shadish, Cook \& Campbell, 2002).

Participants' preferences for treatment have been identified as a deterrent to enrollment in randomized clinical trials (RCTs). Eligible persons may decline enrollment in a RCT because of strong preferences for the treatment options under evaluation. They are not willing to take the risk of being randomly assigned to the least preferred option (McPherson \& Britton, 2001; TenHave, Coyne, Salzer \& Katz, 2003). Low enrollment rate of persons with strong preferences results in a sample size which is smaller than required to attain adequate statistical power. Further, persons with preferences form a subgroup of the target population; if they decline entry into the RCT, then the obtained sample may not be representative of all subgroups comprising the population (Howard \& Thornicroft, 2006; Milat et al., 2005), thereby limiting generalizability of findings (Leykin et al., 2007).

The partially randomized clinical trial (PRCT) or preference trial has been proposed as an alternative design for intervention evaluation research to increase enrollment rate and accrual of the required sample size, and to enhance sample representativeness (TenHave et al., 2003). In the PRCT, participants' preferences are taken into account when allocating them to treatment. Participants indicating a preference are allocated to the intervention of their choice, whereas those with no preference are randomly assigned to the interventions under evaluation (Preference Collaborative Review Group, PCRG, 2009). Although the PRCT has the potential to overcome the limitations of the RCT, there is limited empirical evidence supporting the identified advantages of the PRCT in improving enrollment rate and sample representativeness. These advantages are of particular relevance in the evaluation of behavioral treatments for the management of chronic insomnia. RCTs of such behavioral interventions are characterized by low participation rates yielding small sample sizes (Morin et al., 2006).

The purpose of this methodological multi-site study was to determine the extent to which the PRCT, as compared to the RCT, design increases enrollment of eligible persons in the study and enhances representativeness of the accrued sample. A large-scale methodological study described elsewhere (Sidani et al., 2007) involved two sites, one of which was committed to an RCT design, the other following the specifications for a PRCT. The specific objectives were: 1) to examine enrollment rates in the site that implemented the RCT and the site that implemented the PRCT design; 2) to report reasons given by persons for declining participation in the RCT and PRCT trials; and 3) to describe the clinical characteristics related to chronic insomnia reported by persons 
TREATMENT PREFERENCES AND ENROLLMENT

who declined participation and those who enrolled in the study, in the context of RCT and PRCT designs.

\section{Relevant Literature}

In this section, available empirical evidence about enrollment in RCT and PRCT is summarized. Specifically, enrollment rates, reasons for declining participation, and results of comparisons between enrollees and non-enrollees in RCT and PRCT trials are synthesized.

\section{Enrollment pattern in RCT}

Enrollment rates. Typically, a high ( $\geq 80 \%$ ) enrollment rate and conversely, a low refusal rate are required to achieve an adequate sample size and to maintain sample representativeness. Recent trends indicate that at least a quarter of eligible persons decline participation in RCTs evaluating different types of interventions in different target populations. For instance, Jenkins and Fallowfield (2000) found that $25 \%$ of participants declined entry in a RCT of cancer therapy. Similar refusal rates were reported in RCTs that evaluated pharmacological and/or behavioral treatments for chronic insomnia. J acobs et al. (2004) reported that $27 \%$ of eligible participants declined enrollment in a RCT evaluating the effectiveness of cognitive behavior therapy and sleep medication in middle-aged adults with insomnia. In other studies aimed at determining the effects of cognitive behavior therapy only, the percentage of eligible persons who declined enrollment ranged between 26\% and 45\% (Edinger et al., 2007; Pallesen et al., 2003; Savard, Simrad, Ivers \& Morin, 2005).

Reasons for refusal. Multiple and diverse reasons for declining enrollment are given by persons eligible for participation in RCTs. The most frequently mentioned reasons are categorized into factors related to the participants and to the study design. Participant-related factors include personal, psychological, or lifestyle characteristics that may influence the individuals' decision to enroll in the study. Examples of these characteristics are employment status; transportation difficulties; misperception or negative attitude toward research, which tends to be prevalent among some racial / ethnic groups; safety concerns and poor health status, which are often cited by older persons; lack of time or interest in the study topic; and fear of loss of health benefits (Harris \& Dyson, 2001; Heaman, 2001; J uion, Gross \& Barclay-McLaughlin, 2000; Kalafut DiMattio, 2001). Concerns about the study design also appear to prevent eligible persons from enrolling in a RCT. Persons voice concerns about being treated as a "guinea pig" or experimental subject (Stevens \& Ahmedzai, 2004). They resent randomization; they feel randomization is unfair and decreases their sense of control. Participants often express a 
desire to be actively involved in treatment-related decision making or to leave treatment decision to their physician (J enkins \& Fallowfield, 2000; Stevens \& Ahmedzai, 2004). The percentage of participants who accept random assignment to treatment options was estimated to range between 26\% and 88\% across studies (King et al., 2005).

Participants' preferences for treatment are emerging as an important reason for non-enrollment in a RCT (Thomas et al., 2004). Persons with a preference for a particular intervention opt to not participate in a RCT to avoid the risk of being randomly assigned to the treatment of least preference. Stevens and Ahmedzai (2004) reported that participants who viewed the intervention under evaluation as "not proper treatment" declined enrollment in a RCT of adjuvant therapy for breast cancer. Oakley et al. (2003) found that 5 (10\%) of 49 schools showing initial willingness to take part in a RCT of peer-led sex education withdrew from the study because of random assignment to the non-preferred intervention. Klaber Moffett et al. (2004) reported that 45\% of eligible participants for a RCT evaluating the effectiveness of a brief physiotherapy intervention were not willing to be randomized and refused entry into the RCT because of treatment preferences. Macias et al. (2005) reported that 30\% of eligible persons declined participation because they did not want to risk assignment to the non-preferred intervention for managing mental health problems.

Comparison between enrollees and non-enrollees. The findings presented in the previous section suggest that participants in a RCT may differ from those declining entry in a RCT in terms of treatment preferences. As compared to participants, non-enrollees have clear preferences for the interventions under evaluation and are not willing to be randomized. There is empirical evidence indicating that persons with preferences differ from persons with no preferences for treatment in their socio-demographic profile. For instance, Dwight-J ohnson et al. (2001) found that enrollees in a RCT comparing medication and psychotherapy for depression differed from non-enrollees. Enrollees had higher levels of education, were more likely to be female and older, and more often expressed preferences for the treatments under evaluation. In a metaanalysis of PRCTs, King et al. (2005) found that participants with treatment preferences were more likely than those with no preferences to be women, well-educated, white, and employed. The observed differences between enrollees and non-enrollees in a RCT on socio-demographic characteristics and preferences compromise the represetativeness of the sample and limit the generalizability of the RCT findings to all groups comprising the target population (Leykin et al. 2007; Milat et al., 2005). 


\section{Enrollment pattern in PRCT}

Enrollment rate. The PRCT is characterized by accounting for participants' preferences when allocating them to treatment (TenHave et al., 2003). Accounting for treatment preferences is appealing to persons who would have refused enrollment for fear of receiving the non-preferred treatment. This strategy is believed to overcome the barrier to enrollment (related to preferences) in a RCT, thereby increasing participation rate and enhancing sample representativeness of all subgroups comprising the target population (McPherson \& Britton, 2001). The limited available empirical evidence is inconclusive in supporting this claim. J anevic et al. (2003) examined enrollment rate in the randomized and preference arms of the trial aimed at evaluating two educational interventions for the management of heart diseases. Enrollment rate did not differ between the two arms; it was 38\% in the preference and 36\% in the random arm. Awad et al. (2000) reported a significant difference in the percentage of persons with endulism (i.e., loss of teeth) who declined entry into treatment, based on method of assignment to treatment. Refusal rates were $3 \%$ in the preference arm and 30\% in the randomized arm of the trial. King et al. (2005) conducted a systematic review of 23 trials that accounted for participants' preferences when allocating them to various medical or surgical interventions. The results showed that enrollment rates ranged from $50 \%$ to $100 \%$. The authors concluded that accounting for preferences reduced recruitment issues by increasing enrollment.

Comparison between enrollees and non-enrollees. No study was found that examined differences in personal and clinical characteristics between enrollees and non-enrollees in a PRCT. The results of such a comparison would determine comparability of these two subgroups of the target population and, hence, representativeness of the sample. In two studies mentioned in the previous section, participants in the preference and the randomized arms were compared on baseline characteristics to determine the extent of self-selection bias introduced in the preference arm. J anevic et al. (2003) found no significant differences in the socio-demographic and health characteristics of participants in the two arms. The results of King et al.'s (2005) meta-analysis indicated that participants in the randomized and preference arms differed on at least one characteristic; such differences were reported in 9 of 20 trials. It is unclear if the inconsistency in the findings of these two studies is due to differences in the target populations or the types of treatments under evaluation, i.e., educational (J anevic et al.) versus medical and surgical (King et al.). The extent to which allocation to treatments of preference enhances enrollment rate and sample representativeness in studies evaluating behavioral interventions is not known ( $\mathrm{J}$ anevic et al.). This 
knowledge gap was addressed in this study in which behavioral treatments for chronic insomnia were investigated.

\section{Method}

\section{Design}

This study was conducted in two sites that implemented different research designs but the same behavioral interventions for managing insomnia and used the same eligibility criteria for selecting participants. In site 1, a RCT design was used. Participants were randomly assigned to the experimental or comparison treatment. In addition, polysomnography (PSG) assessment was planned as part of baseline data collection. The incorporation of PSG is consistent with the design of other RCTs evaluating interventions for insomnia, and is done to assess the presence of sleep disorders (Buysse et al., 2006). In site 2, consistent with the PRCT design, participants indicated their preference for either the experimental or comparison treatment. Participants with a preference were allocated to the intervention of their choice, while those with no preference were randomized to treatment. No baseline PSG assessment was done in this site for logistical reasons.

The experimental treatment was a multi-component intervention (MCI), which consisted of sleep education and hygiene, stimulus control instructions, and sleep restriction therapy. The comparison treatment entailed sleep education and hygiene (SEH) only.

The data for this methodological study were gathered during the initial research steps undertaken to determine eligibility and interest in taking part in the study. The data were obtained from persons who orally consented to complete the screening questionnaire and who met the selection criteria related to age, English language proficiency, and clinical characteristics of insomnia (i.e., type, frequency, and duration).

\section{Sample}

The target population consisted of persons with complaint of chronic insomnia. Persons were eligible to participate in the study if they 1) were community-dwelling, non-institutionalized adults 21 years of age or older; 2) were able to read and write English; and 3) complained of difficulty initiating and/or maintaining sleep, manifested by time to fall asleep and/ or time awake after sleep onset of 30 minutes or more per night, and experienced for a minimum of three nights per week, for a duration of at least three months. The required sample size was 100 in the RCT design and 200 in the PRCT design. The difference in the total number of participants accounted for the costs and logistics of conducting baseline 
PSG assessment in the RCT design, and for the need to balance the distribution of participants across the randomized and preference subgroups in the PRCT design.

\section{Variables and Measures}

Enrollment and refusal rates. Enrollment rate refers to the percentage of persons meeting the initial eligibility criteria who agree to take part in the study (Des Jarlais, Lyles, Crepaz \& the TREND group, 2004). For the purpose of this study, enrollment rate was computed as the percentage of persons who consented to initial screening, met the eligibility criteria, and continued their participation in the study; that is, they agreed to move through the next step of the study protocol that consistent of completing the pretest measures. Refusal rate represented the percentage of eligible persons who declined further involvement in the study, that is, they did not complete the pretest measures. At each study site, the research assistant kept a log documenting the following for each person who called the research office to inquire about the study: provided verbal consent to respond to the screening questionnaire; met eligibility criteria; and indicated refusal to take part or expressed interest in continued participation in the study.

Reasons for refusal. The research assistant requested participants who declined further enrollment in the study to indicate the reason(s) for refusal. The research assistant wrote down, verbatim, the information shared by participants, and documented 'no reason given' when participants did not provide any explanation for their decision.

Screening questionnaire. In addition to living condition (i.e., community-dwelling), age, English language proficiency, the screening questionnaire assessed clinical characteristics of insomnia to determine eligibility. The clinical characteristics reflected participants' experience of insomnia in terms of type, frequency, and duration, which were used to assess sample representativeness. The investigators developed pertinent open and close-ended questions and response options to gather data on the following specific indicators of insomnia: 1) type of insomnia: participants indicated whether or not they had difficulty falling asleep and/or difficulty staying asleep; 2) sleep onset latency: participants reported the average length, in minutes, to fall asleep per night, 3) wake after sleep onset: participants reported the average number of times awake and the average length of time, in minutes, awake during a night; 4) average amount of sleep, in minutes, per night; 5) average number of nights per week during which insomnia is experienced; 6) duration (in years and month) of insomnia; and 7) whether or not insomnia is viewed as bothersome. 


\section{Procedure}

The study protocol was approved by the Institutional Review Board at the participating institution at each site. The same procedures for recruitment and assessment of the clinical characteristics of insomnia were followed in the two sites. Persons with insomnia were recruited through advertisement in newspaper and newsletters; distribution of flyers to community centers, health clinics, and specialized sleep clinics; referral by health care providers; and radio announcement. Persons interested in the study were requested to call the research office. During the phone call, the research assistant explained the study requirements and the incentive (\$40) offered upon study completion, and described briefly the treatment options offered and the method of assignment to treatment options at the respective site. After obtaining participants' oral consent, the research assistant administered the screening questionnaire assessing their eligibility based on age, English language proficiency, and clinical characteristics of insomnia. The research assistant then inquired about their interest in continued participation in the study. Persons declining further enrollment were requested to indicate the reason(s) for refusal.

\section{Data Analysis}

Objective 1. Descriptive statistics were used to determine the enrollment and refusal rates for each participating site.

Objective 2. The reasons given by participants for declining enrollment were content analyzed. Because some participants provided more than one reason, the number of times a particular reason was mentioned was reported.

Objective 3. Chi-square test or independent sample t-test, based on the variable's level of measurement, was used to compare eligible persons who declined participation (non-enrollees) and persons who enrolled in the study (enrollees) on the clinical characteristics of insomnia within each site. An appropriate formula was used when the equality of variance assumption for the t-test was not achieved. The level of statistical significance was set at $\mathrm{p} \leq .05$. In addition, the magnitude of the differences between enrollees and non-enrollees on the insomnia characteristics was assessed. This analysis was conducted because some of the observed statistically significant differences appeared too small to be clinically meaningful. The magnitude of the differences was estimated for continuous variables, using the effect size. The effect size was computed as the standardized mean differences between enrollees and non-enrollees. 


\section{Results}

The results are presented for each site to show the enrollment rate, reasons for refusal, and comparability of enrollees and non-enrollees under the RCT and PRCT designs. There were not statistically significant between-site differences in the clinical characteristics of insomnia.

\section{Site 1 - RCT design}

The study protocol in site 1 involved randomization to treatment. A total of 211 persons provided oral consent to respond to the screening questionnaire and met the study eligibility criteria.

Enrollment rate. Of the 211 eligible persons, 195 enrolled and 16 declined further participation in the study. The enrollment rate was $92.4 \%$ and the refusal rate was $7.6 \%$.

Reasons for refusal. Participants gave multiple reasons for declining to take part in the study. These were related to characteristics of participants and characteristics of the study. Characteristics of participants entailed "lack of interest in the study" (representing 79\% of the reasons mentioned), "too busy" (7\%), and "not feeling well enough" (2\%). Characteristics of the study that deterred enrollment were: "don't want overnight" (i.e., spending a night at the sleep laboratory to undergo PSG assessment; 7\%), and "study is time intensive" (5\%).

Table 1

Clinical characteristics of enrollees and non-enrollees in site 1 using RCT design

\begin{tabular}{lccr}
\hline Characteristic & $\begin{array}{c}\text { Enrollees } \\
(\mathrm{n}=195)\end{array}$ & $\begin{array}{l}\text { Non-enrollees } \\
(\mathrm{n}=16)\end{array}$ & Difference \\
\hline Type of insomnia: & & & \\
$\quad$ Difficulty falling asleep (\%) & 70 & 75 & \\
$\quad$ Difficulty staying asleep (\%) & 94 & 94 & \\
Minutes to fall asleep (M, SD) & $50(50)$ & $53(38)$ & -0.06 \\
Times awake during a night (M, SD) & $3(1.5)$ & $3.5(2.2)$ & -0.33 \\
Minutes awake during a night (M, SD) & $103(67)$ & $136(85)$ & -0.48 \\
Hours of sleep per night (M, SD) & $5.2(1.3)$ & $4.7(1.5)$ & 0.38 \\
Nights/ week with insomnia (M, SD) & $5.9(1.4)$ & $5.1(1.4)$ & 0.61 \\
Duration (years) of insomnia (M, SD) & $11.1(10.1)$ & $6.7(6.8)$ & 0.44 \\
Insomnia viewed as bothersome (\%) & 98 & 100 & \\
\hline
\end{tabular}

$* \mathrm{p} \leq .05$

Comparison between enrollees and non-enrollees. Table 1 presents measures of central tendency and dispersion for enrollees and non-enrollees in the RCT, as well as the effect sizes reflecting the magnitude of the differences between the two groups on continuous variables. A statistically significant between-group difference was found 
for one variable only: number of nights per week during which insomnia is experienced $(\mathrm{t}(191)=2.14, \mathrm{p}=.03)$. Enrollees reported a larger number of nights with insomnia than did non-enrollees. This difference was of a moderate-to-high magnitude. Although the between-group differences for the remaining insomnia characteristics were not statistically significant, the respective effect sizes suggested a low-to-moderate differences between enrollees and non-enrollees in: number of times awake during a night, length of time awake during a night, amount of sleep per night, and duration of insomnia. Overall, the mean values on these characteristics imply that enrollees tended to have insomnia of lower level of severity but of longer duration than non-enrollees.

\section{Site 2 - PRCT design}

The study protocol in site 2 entailed allocating participants to the behavioral intervention of their preference. A total of 493 persons verbally consented to complete the screening questionnaires and met the initial eligibility criteria.

Enrollment rate. Of the 493 eligible persons, 221 enrolled and 272 did not enroll in the study. The enrollment rate was $44.8 \%$ and the refusal rate was $55.2 \%$.

Reasons for refusal. The reasons for declining enrollment related to characteristics of participants, characteristics of the study, and logistics. Characteristics of participants included "lack of interest in the study" (46\%), "too busy" (8\%), and "found out the reason for insomnia" (8\%). The study characteristics mentioned as reasons for non-participation were: "compensation is not enough" (23\%) and "study time is intensive" (10\%). Logistics related to "travel distance to study site" was mentioned by $15 \%$ of non-enrollees as the reason for not taking part in the study.

Comparison between enrollees and non-enrollees. The average values for enrollees and non-enrollees at site 1 are presented in Table 2. Statistically significant differences between the two groups were found for four characteristics of insomnia: difficulty falling asleep $\left(\chi^{2}(1)=4.29, \mathrm{p}=\right.$ $.03)$, length of time to fall asleep $(\mathrm{t}(491)=3.23, \mathrm{p}=.001)$, length of time awake during the night $(\mathrm{t}(476)=2.12, \mathrm{p}=.03)$, and amount of sleep per night $(\mathrm{t}(491)=2.79, \mathrm{p}=.005)$. While the differences were of a small magnitude (all effect sizes $\leq .30$ ), they portrayed the following profile of enrollees as compared to non-enrollees: less frequent experience of difficulty falling asleep with a shorter time to fall asleep and length of time awake during a night, but longer amount of sleep. Overall, enrollees appear to experience insomnia of lower level of severity than that reported by non-enrollees. 


\section{TREATMENT PREFERENCES AND ENROLLMENT}

Table 2

Clinical characteristics of enrollees and non-enrollees in site 2 using PRCT

design

Characteristic

Type of insomnia:

Difficulty falling asleep (\%)*

Difficulty staying asleep (\%)

Minutes to fall asleep (M, SD)*

Times awake during a night (M, SD)

Minutes awake during a night (M, SD)*

Hours of sleep per night (M, SD)*

Nights/ week with insomnia (M, SD)

Duration (years) of insomnia (M, SD)

Insomnia viewed as bothersome (\%)

$* \mathrm{p} \leq .05$

$\begin{array}{ccr}\begin{array}{c}\text { Enrollees } \\ (\mathrm{n}=221)\end{array} & \begin{array}{c}\text { Non-enrollees } \\ (\mathrm{n}=272)\end{array} & \text { Difference } \\ & & \\ 71.5 & 80 & \\ 93 & 93 & -0.30 \\ 53(64) & 73(68) & -0.12 \\ 2.5(1.8) & 2.7(1.5) & -0.20 \\ 89(65) & 104(82) & 0.27 \\ 5.4(1.2) & 5.1(1.2) & -0.07 \\ 5.8(1.5) & 5.9(1.4) & 0.11 \\ 10.4(10.3) & 9.2(10.6) & \\ 97 & 99 & \end{array}$

\section{Discussion}

The results of this methodological study showed higher enrollment rate in the RCT than the PRCT. Overall, similar reasons for refusal to take part in the study were given by participants in the RCT and the PRCT trials. Statistically significant differences between enrollees and non-enrollees were found on fewer clinical characteristics of insomnia in the RCT than the PRCT. However, clinically meaningful differences between enrollees and non-enrollees were reported on a larger number of insomnia characteristics under the RCT than the PRCT design. The small sample size and the unbalanced number of non-enrollees and enrollees in the RCT could have influenced the power to detect significant differences in the clinical characteristics. Overall, these findings do not provide conclusive evidence supporting the advantages of accounting for participants' treatment preferences in insomnia intervention evaluation research, relative to increased enrollment and enhanced representativeness of the accrued sample.

The enrollment rate was almost twice as high in the site using the RCT (92\%) as in the site implementing the PRCT (45\%) design. This finding differs from the results of the meta-analysis conducted by King et al. (2005), which indicated that accounting for participants' preferences improved enrollment in trials of medical or surgical interventions. The differences in the patient populations and nature of treatments under investigation in this methodological study when compared to the metaanalytic study mean that the high enrollment rate observed in the RCT site should be interpreted with caution. The observed rate is much higher than the average enrollment rate reported in studies of behavioral treatments for insomnia. In contrast, the enrollment rates in the PRCT are 
comparable to those reported in previous research (Edinger et al., 2007; Pallesen et al., 2003; Savard et al., 2005). The exact factors that could have contributed to this pattern of enrollment rates are not clear and require further investigation. Possible across-site differences in the sociodemographic and clinical profile of persons with insomnia targeted through recruitment could be a factor; however, the characteristics assessed in this study were comparable for participants in the two sites. Differences in the beliefs about insomnia and its treatment, and in the values of research could have contributed to differences in enrollment rates between sites, and should be explored in future research. The plan for conducting PSG assessment in the RCT site could have been an attractive feature of the study and encouraged participation; the plausibility of this factor is questionable in light of current findings indicating that PSG assessment deterred enrollment in few cases. Additional exploration of the role that PSG assessment could play in affecting enrollment is informative. The characteristics of the research personnel hired at the two sites represent another factor influencing enrollment rate. Although the same professional qualities were applied to select the research personnel, and the same standard script was followed in the recruitment process at the two sites, differences in the interactional style of research personnel could have affected individuals' participation in the study.

Similar reasons for refusals were given by participants in the two sites. The most frequently mentioned reasons for refusing enrollment were related to characteristics of participants (primarily, lack of interest) and of the study (primarily, study is time intensive). Issues related to compensation and to logistics were raised in the site implementing the PRCT. Apart from the latter reasons, none of the reasons given had to do with preferences for the treatment options under investigation, which in other studies have been found to influence participants' decision to enroll in a RCT (Oakley et al., 2003; Stevens \& Ahmedzai, 2004). Further, nonenrollees in the RCT site did not express concern about random assignment, as was reported in previous research (e.g., Jenkins \& Fallowfield, 2000). Conceptual and methodological factors could explain variability in the reasons for refusal provided by participants in this and previous studies. Conceptually, diversity in the target populations and in the types of treatment investigated across studies could account for the concern about randomization reported in previous studies and not in the present one. The concern was expressed by persons presenting with acute, potentially life-threatening conditions, such as cancer (Stevens \& Ahmedzai, 2004), requiring pharmacological treatment that may be perceived as invasive and associated with side effects. In contrast, persons recruited for this study experienced a chronic, non-life threatening condition, and were offered behavioral interventions with no or minimal 
side effects. From a methodological perspective, variation in study protocol could explain differences in expression of concern about randomization between this and previous studies. In previous studies, persons received detailed information about treatment options and treatment allocation procedure in writing or in a face-to-face meeting with the researcher before indicating their decision to enroll in the study and the reasons for refusal. In this study, the information was given over the telephone, potentially leading to misinterpretation of or lack of clarity about some study details.

The evidence supporting the advantage of the PRCT in enhancing the representativeness of the sample, as gathered in this study, is inconclusive. The evidence was based on the comparisons between enrollees and nonenrollees within the RCT and PRCT designs. The statistical and clinical significance of the between-group differences were examined. In the RCT, the difference was statistically significant $(p \leq .05)$ for one variable; however, it was of a moderate-to-high magnitude for four of the six continuous variables included in the between-group comparisons. In the PRCT, there were statistically significant differences between enrollees and non-enrollees on four of the six variables; yet the differences were of a small-to-moderate magnitude. This pattern of findings could be related to the difference in the sample size obtained in the RCT and the PRCT. The number of participants was smaller in the RCT $(\mathrm{n}=211)$ than the PRCT ( $\mathrm{n}$ $=493$ ). There is a tendency toward reporting spurious large effect sizes with small samples (Lipsey, 1990).

In conclusion, this methodological study is the first to examine the advantage of the PRCT design relative to enrollment rate and sample representativeness. The results do not support the advantage of the PRCT in enhancing enrollment of participants in studies evaluating the effectiveness of behavioral interventions for chronic insomnia. Additional studies are required to further investigate the advantage of the PRCT design in maintaining sample representativeness. Such studies should be carefully designed to accrue a comparable number of participants in the RCT and PRCT, which is necessary for meaningful comparisons between enrollees and non-enrollees in each design.

\section{Acknowledgements}

Funding was provided by the National Institutes of Health - National Institute of Nursing Research (NR05075). Resources were also provided by the Phoenix Veterans Affairs Health Care System. The contents do not represent the views of the Department of Veterans Affairs or the United 
States Government. Souraya Sidani can be reached by e-mail at ssidani@ryerson.ca.

\section{References}

Awad, M.A., Shapiro, S.H., Lund, J.P., \& Feine, J.S. (2000). Determinants of patients' treatment preferences in a clinical trial. Community Dental Oral Epidemiology, 28, 119-125.

Buyesse, D.J., Ancoli-Israel, S., Edinger, J.D., Lichstein, K.L. \& Morin, C.M. (2006). Recommendations for a standard research assessment of insomnia. Sleep, 29, 1155-1173.

Des J arlais, D.C., Lyles, C., Crepaz, N. \& the TREND Group. (2004). Improving the reporting quality of nonrandomized evaluations of behavioral and public health interventions: The TREND statement. American Journal of Public Health, 94, 361-366.

Dwight-J ohnson, M., Unutzer, J., Sherbourne, C., Tang, L., \& Wells, K.B. (2001). Can quality improvement programs for depression in primary care address patient preferences for treatment? Medical Care, 39 (9), 934-944.

Edinger, J.D., Wohlgemuth, W.K., Radtke, R.A., Coffman, C.J. \& Carney, C.E. (2007). Dose-response effects of cognitive-behavioral insomnia therapy: A randomized clinical trial. Sleep, 30 (2), 203-212.

Harris, R., \& Dyson, E. (2001). Recruitment of frail older people to research: Lessons learnt through experience. Journal of Advanced Nursing, 36 (5), 643-651.

Heaman, M. (2001). Conducting health research with vulnerable women: Issues and strategies. Canadian J ournal of Nursing Research, 33 (3), 81-86.

Howard, L. \& Thornicroft, G. (2006). Patient preference and randomized controlled trials in mental health research. British Journal of Psychiatry, 188, 303-304.

Jacobs, G.D., Pace-Schott, E.F., Stickgold, R. \& Otto, M.W. (2004). Cognitive behavior therapy and pharmacotherapy for insomnia. Archives of Internal Medicine, 164, 1888-1896.

J anevic, M.R., Janz, M.K., Dodge, J.A., Lin, X., Pan, W., Sinco, B.R., \& Clark, N.M. (2003). The role of choice in health education intervention trials: A review and case study. Social Science \& Medicine, 56, 1581-1594.

J enkins, V. \& Fallowfield, L. (2000). Reasons for accepting or declining to participate in randomized clinical trials for cancer therapy. British J ournal of Cancer, 82(11), 1783-1788.

J ulion, W., Gross, D., \& Barclay-McLaughlin, G. (2000). Recruiting families of color from the inner city: Insights from recruiters. Nursing Outlook, 48 (5), 230-237.

Kalafut DiMattio, M.J. (2001). Recruitment and retention of communitydwelling, aging women in nursing studies. Nursing Research, 50 (6), 369373.

King, M., Nazareth, I., Lampe, F., Bower, P., Chandler, M., Morou, M., Sibbald, B., \& Lai, R. (2005). Impact of participant and physician intervention preferences on randomized trials. A systematic review. JAMA, 293, 1089-1099. 


\section{TREATMENT PREFERENCES AND ENROLLMENT}

Moffett, J. A. K., Jackson, D. A., Richmond, S., Hahn, S., Coulton, S., \& et al. (2004). Randomized trial of a brief physiotherapy intervention compared with usual physiotherapy for neck pain patients: Outcomes and patients' preference. BMJ Online, 10, 1-6.

Lambert, M.F. \& Wood, J. (2000). Incorporating patient preferences into randomized trials. J ournal of Clinical Epidemiology, 53, 163-166.

Leykin, Y., DeRubeis, R.J ., Gallop, R., Amsterdam, J.D., Shelton, R.C. \& Hollon, S.D. (2007). The relation of patients' treatment preferences to outcome in a randomized clinical trial. Behavior Therapy, 38, 209-217.

Lipsey, M. W. (1990). Design sensitivity: Statistical power for experimental research. Newburt Park, CA: Sage.

Macias, C., Barreira, P., Hargreaves, W., Bickman, L., Fisher, W., \& Aronson, E. (2005). Impact of referral source and study applicants' preference for randomly assigned service on research enrollment, service engagement, and evaluative outcomes. American J ournal of Psychiatry, 162 (4), 781-787.

McPherson, K. \& Britton, A. (2001). Preferences and understanding their effects on health. Quality in Health Care, 10, i61-i66.

Millat, B., Borie, F. \& Fingerhut, A. (2005). Patients' preference and randomization: New paradigm of evidence-based clinical research. World J ournal of Surgery, 29, 596-600.

Morin, C. M., Bootzin, R. R., Buysse, D. J., Edinger, J. D., Espie, C. A., \& Lichstein, K. L. (2006). Psychological and behavioral treatment of insomnia: Update of the recent evidence (1998-2004). Sleep, 29, 1398-1414.

Oakley, A., Strange, V., Toroyan, T., Wiggins, M., Roberts, I. \& Stephenson, J . (2003). Using random allocation to evaluate social interventions: Three recent U.K. examples. Annals of the AAPSS, 589, 170-189.

Pallesen, S., Nordhus, I.H., Kvale, G., Nielsen, G.H., Havik, O.E., J ohnsen, B.H. \& Skjoskift, S. (2003). Behavioral treatment of insomnia in older adults: An open clinical trial comparing two interventions. Behavior Research \& Therapy, 41, 31-48.

Preference Collaborative Review Group (2009). Patients' preferences within randomized trials: Systematic review and patient level meta-analysis. British Medical J ournal, 338, a1864

Savard, J ., Simard, S., Hervouet, S., Ivers, H., Lacombe, L., \& Fradet, Y. (2005). Insomnia in men treated with radical prostatectomy for prostate cancer. Psycho-Oncology, 14, 147-156.

Shadish, W.R., Cook, T.D., \& Campbell, D.T. (2002). Experimental and quasiexperimental design for generalized causal inference. Boston, MA: Houghton-Mifflin.

Sidani, S., Epstein, D.R., Bootzin, R.R., Moritz, P. \& Sechrest, L. (2007). Alternative methods for clinical research (NP 05075). Final report submitted to the National Institute of Nursing Research, Washington, DC.

Stevens, T. \&Ahmedzai, S.H. (2004). Why do breast cancer patients decline entry into randomized trials and how do they feel about their decision later: A prospective, longitudinal, in-depth interview study. Patient Education \& Counseling, 52, 341-338.

TenHave, T.R., Coyne, J., Salzer, M., \& Katz, I. (2003). Research to improve the simple randomized clinical trial. General Hospital Psychiatry, 25, 115-123. 
Thomas, E., Croft, P.R., Paterson, S.M., Dziedzic, K., \& Hay, E.M. (2004). What influences participants' treatment preference and can it influence outcome? Results from a primary care-based randomized trial for shoulder pain. British J ournal of General Practice, 54, 93-96. 